\title{
A Comparative Evaluation of Risk Factors and Various Antibiotic Regimens in Postoperative Neurosurgical Infections: A Prospective Randomized Controlled Study
}

\author{
R. Shetty ${ }^{1}$ \\ S. Dwarakanath ${ }^{1}$ \\ H.B. Veena Kumari² \\ ${ }^{1}$ Department of Neurosurgery, National Institute of Mental Health and \\ Neurosciences (NIMHANS), Hosur Road, Bengaluru, Karnataka, India \\ 2 Department of Neuromicrobiology, National Institute of Mental \\ Health and Neurosciences (NIMHANS), Hosur Road, Bengaluru, \\ Karnataka, India
}

Indian J Neurosurg 2017;6:163-169.

\author{
S. Sampath ${ }^{1}$ \\ B.A. Chandramouli ${ }^{1}$
}

\begin{abstract}
Address for correspondence Dr. S. Dwarakanath, MS, MCh, Department of Neurosurgery, NIMHANS, Bengaluru, Karnataka 560029, India (e-mail: dwarakaneuro@yahoo.com).
\end{abstract}

\begin{abstract}
Keywords

- antibiotics

- infections

- cefazolin

- Amoxicillinclavulanic acid

- cefotaxime

Introduction Postoperative neurosurgical infections are a major problem, and the importance of keeping them to an absolute minimum cannot but be overemphasized. In this prospective study, we plan to evaluate the efficacy of three antibiotic regimens designed keeping the availability, affordability, and efficacy upon the common bacterial flora isolated at our center. We will also analyze the incidence and evaluate the risk factors of surgical site infections following clean, nonimplant, fresh, elective craniotomies.

Material and Methods This prospective randomized control study was conducted at our institute, a tertiary referral center, for a period of 2 years. The study cohort included all patients undergoing clean, nonimplant, fresh, elective craniotomies at our institute. The antibiotic protocol was chosen according to the current bacterioepidemiological data based on the prevalence of infectious agent. One of the three drug regimens was chosen.

Results The results of univariate analysis revealed that there are multiple factors that are significantly associated with postoperative surgical site infection. In the multivariate analysis, only the presence of cerebrospinal fluid leak was the most significant factor associated with postoperative infections. Among the three different antibiotic regimens, cefazolin and amoxicillin-clavulanic acid were much better prophylactic agents for preventing surgical site infections when compared with cefotaxime.

Conclusion The present study has provided an insight into the incidence and risk factors contributing to surgical site infections as well as the efficacious antibiotic regimen protocols. The present study is one of the few studies to compare the efficacy of different antibiotic regimen protocols and also prospectively analyze the factors contributing to postoperative infections. This study will help us in optimizing the prophylaxis protocols and prevent emergence of multidrug-resistant strains, especially in a country like India.
\end{abstract}

received

November 21, 2015

accepted

January 18, 2016

published online

October 3, 2017
DOI https://doi.org/

10.1055/s-0036-1581977. ISSN 2277-954X. (c) 2017 Neurological Surgeons' Society License terms of India

(c) $(1) \$$ 


\section{Introduction}

Postoperative neurosurgical infections are a major problem, and the importance of keeping them to an absolute minimum cannot but be overemphasized. The morbidity and mortality incurred by the patients and the financial burdens that they impose on the health care delivery system are potentially significant. The prophylactic use of antibiotics has been suggested as a way of preventing infection but the subject remains controversial. However, since the landmark study of Malis at the University of Pittsburgh in 1979, who reported the absence of infection in 1,732 major clean operative cases by using antibiotic prophylaxis, neurosurgeons all over the world routinely use prophylactic antibiotics prior to craniotomies. At present, although the role of prophylactic antibiotics has been established, there remains a disagreement on the nature and duration of antibiotic usage. Various institutions follow different prophylactic regimens depending upon the cost and efficacy of antibiotics and the prevalence of the strains of bacteria.

\section{Background of the Current Study}

Systemic antibiotic prophylaxis effectively reduces the rate of infection following clean neurosurgical operations by approximately fourfold. The intent of this study was to emphasize the fact that there is a rise in antibiotic resistance and to remind physicians about their role in society toward appropriate and judicious use of antibiotics. The predominance of studies in neurosurgery has demonstrated efficacy of prophylactic antibiotics, indicating that further testing of this procedure against placebo controls is unethical. It may, however, be necessary and ethical to conduct trials comparing different antibiotic regimens.

\section{Rationale behind Current Study}

India is a developing country, and the majority of our patient input comes from poor socioeconomic strata. The idea was to come up with the best antibiotic regimen according to the institutional needs, weigh the cost-benefit ratio including drug side effects, and prevent the emergence of antibioticresistant strains of organisms. In this prospective study, we plan to evaluate the efficacy of three antibiotic regimens designed keeping the availability, affordability, and efficacy upon the common bacterial flora isolated at our center.

\section{Rationale behind Selection of Three Antibiotic Regimens}

Even though the previous study done in our institution showed that the microepidemiologic data indicated predominantly gram-negative organisms in surgical site infections, recent data have indicated rise in gram-positive organisms, especially staphylococcal species, in causing postoperative surgical site infections. Hence, this study was designed to use $\beta$-lactam antibiotics, which are active against gram-positive organisms, and to compare the efficacious role of these antibiotics.

\section{Aims and Objectives}

- To determine the incidence and evaluate the risk factors of surgical site infections following clean, nonimplant, fresh, elective craniotomies

- Evaluate the efficacy of antibiotics in patients treated with three different antibiotic regimen protocols

\section{Material and Methods}

This prospective randomized control study was conducted at our institute, a tertiary referral center, for a period of 2 years from January 2011 to December 2012. The study cohort included all patients undergoing clean, nonimplant, fresh, elective craniotomies at our institute. The criteria for diagnosing and classifying infection were based on the Centre for Disease Control (CDC) ${ }^{1}$ guidelines. The antibiotic protocol (ABP) was chosen according to the current bacterioepidemiological data based on the prevalence of infectious agent. One of the three drug regimens, as mentioned in - Table 1, was chosen. These antibiotics were randomized to patients undergoing clean, nonimplant, fresh, elective craniotomies and are administered after test dose to avoid adverse sensitivity reactions.

Clinico-microbiological assessment: Patients were assessed clinico-neuromicrobiologically as per CDC criteria and follow-up was performed for up to 1 month following surgery or as soon as a surgical site infection was suspected.

\section{Statistical Analysis}

Univariate Analysis

Data were expressed as mean \pm standard deviation. Contingency tables were used for categorical variables and, after categorization, for continuous variables. Chi-square (with Yates correction if needed) and Student $t$-test were used whenever appropriate. All $p$-values less than 0.05 were considered significant.

\section{Multivariate Analysis}

The main purpose of the multivariate analysis was to study the effect of the ABP on patients' infection risk independently of other risk factors. To study the role of the risk factors in the group without "predictive risk factors" and the group with "at least one predictive risk factor," the interactions between "potential risk factors" and the groups was analyzed. Using three-step regression analysis, it was possible to test the stability of the effect of each potential risk factor over time. The models were compared using the likelihood ratio test. Multivariate analysis was performed using the SAS software (SAS software, SAS Institute, Inc., Carey, North Carolina, United States).

\section{Results}

There were a total of 750 patients included in the study. Each of the three arms had 250 patients each. Surgical site infections were seen in 9 of the 750 patients (1.2\%) who underwent clean craniotomies (-Table 2). 
Table 1 Various drug regimens

\begin{tabular}{|l|l|l|l|}
\hline Antibiotic & Dosage and frequency & Repeat dose & Cost (in rupees) \\
\hline $\begin{array}{l}\text { Cefazolin: first-generation } \\
\text { cephalosporin }\end{array}$ & $\begin{array}{l}\text { Adults: } 1 \mathrm{gm} \text {; children: } 25 \mathrm{mg} / \mathrm{kg} ; \\
\text { IV, maximum dose } 1 \mathrm{~g} \\
\text { Administered } 1 \mathrm{~h} \text { prior to } \\
\text { induction }\end{array}$ & $\begin{array}{l}\text { Adults: } 1 \mathrm{~g} ; \text { children: } 25 \mathrm{mg} / \mathrm{kg} ; \\
\text { every } 6 \mathrm{~h} \text { intraoperatively } \\
\text { followed by two doses every } 8 \mathrm{~h} \\
\text { postoperatively }\end{array}$ & 37 \\
\hline $\begin{array}{l}\text { Cefotaxime: third-generation } \\
\text { cephalosporin }\end{array}$ & $\begin{array}{l}\text { Adults: } 1 \mathrm{~g} ; \text { children: } 50 \mathrm{mg} / \mathrm{kg} ; \\
\text { IV, maximum dose } 1 \mathrm{~g} \\
\text { Administered } 1 \mathrm{~h} \text { prior to } \\
\text { induction }\end{array}$ & $\begin{array}{l}\text { Adults: } 1 \mathrm{~g} ; \text { children: } 50 \mathrm{mg} / \mathrm{kg} ; \\
\text { every } 6 \mathrm{~h} \text { intraoperatively } \\
\text { followed by two doses every } 8 \mathrm{~h} \\
\text { postoperatively }\end{array}$ & 35 \\
\hline $\begin{array}{l}\text { Amoxicillin-clavulanic acid: a } \\
\text { penicillin derivative }\end{array}$ & $\begin{array}{l}\text { Adults: } 1.2 \mathrm{~g} \text {; children: } 40 \mathrm{mg} / \mathrm{kg} ; \\
\text { IV, maximum dose } 1.2 \mathrm{~g} \\
\text { Administered } 1 \mathrm{~h} \text { prior to } \\
\text { induction }\end{array}$ & $\begin{array}{l}\text { Adults: } 1.2 \mathrm{~g} \text {; children: } 40 \mathrm{mg} / \mathrm{kg} ; \\
\text { every } 6 \text { h intraoperatively followed } \\
\text { by two doses every } 8 \mathrm{~h} \\
\text { postoperatively }\end{array}$ & 90 \\
\hline
\end{tabular}

Note: These antibiotics were administered in three doses preoperatively. First dose was administered at the time of induction to achieve maximum tissue levels during skin incision. Subsequent doses were given every 8 hours postoperatively according to the bioavailability, half-life, and duration of action. Intraoperative additional doses were given every 6 hours depending upon the duration of surgery.

\section{Assessment of Risk Factors of Surgical Site Infections}

The following factors were analyzed to assess their role in the development of surgical site infections.

Age and sex: There was no statistically significant difference in infection rates in the various decades. Even though the distribution of infection rates was more in female gender, no statistical significance was reached.

Smoking: There were 50 smokers in the study population, one of whom developed surgical site infection; however, no statistical significance in terms of its role in infection was attained.

Diabetes mellitus: Study population had 24 diabetics, none of whom developed surgical site infection. This was statistically significant.

Preoperative hospital stay: The infection rate in the patients who stayed in the hospital for less than a week was $1 \%$, and for those who stayed longer than a week it was $1.3 \%$. The difference was not statistically significant.

Surgical diagnosis: The infection rates in arteriovenous malformation, moyamoya disease, and epilepsy surgery were $7.1 \%(p=0.041), 20 \%(p=0.000)$, and $8.3 \%(p=0.023)$, respectively, which showed statistically significant value.

Table 2 Total number of surgical site infections and details $(N=9)$

\begin{tabular}{|l|l|}
\hline Type of infection & Number of infection \\
\hline Scalp infection & 5 \\
\hline Bone flap osteitis & 3 \\
\hline Subdural empyema & - \\
\hline Brain abscess & - \\
\hline Meningitis & 1 \\
\hline Ventriculitis & - \\
\hline Total & 9 \\
\hline
\end{tabular}

The details of the surgical diagnosis and analysis are presented in - Table 3.

Perioperative corticosteroid administration: The infection rates in patients who received steroids was $0.8 \%$ and those who did not was $7.6 \%$. The infection rates in patients who did not receive steroids were statistically significant.

Head shave: No infection was seen in patients who underwent local head shave; however, it did not reach statistical significance when compared with patients who underwent a complete head shave.

Number of people scrubbed: No infection was seen when only two people had scrubbed for the case, while the infection rates when three or four people had scrubbed reached statistical significance, proving that more the number of people involved in surgery, the greater the chance of infection (-Table 4).

Duration of surgery: In surgeries that finished within 4 hours, infection rate was less, whereas in those with more than 4 hours infection rates were high (1 and 8 , respectively). This was statistically significant.

Wound closure: No statistical significance was reached in terms of the type of wound closure, suture material used, and the incidence of postoperative surgical site infection.

Cerebrospinal fluid leak: The presence of cerebrospinal fluid leak following surgery was statistically highly significant in terms of contributing to postoperative infection (-Table 5).

Preoperative altered sensorium: The state of sensorium of the patient either preoperatively or postoperatively did not contribute to the incidence of surgical site infections.

Multivariate analysis: The results of univariate analysis revealed that there are many factors that are significantly associated with postoperative surgical site infection. It was felt that it is very important to find out the relative importance of each one of these factors' contribution to the infection. Hence, a multivariate analysis was undertaken to find out the variable contributing the most to surgical site infections. It was calculated using odds ratio. Using the above method, it was found that the presence of cerebrospinal fluid 
Table 3 Surgical diagnosis and infection frequency

\begin{tabular}{|l|l|l|l|l|l|}
\hline Diagnosis & Noninfected & Infected & Total & $p$-Value & Significance \\
\hline Glial tumors & 282 & $2(0.7 \%)$ & 284 & 0.443 & No \\
\hline Other tumors & 83 & - & 83 & 0.315 & No \\
\hline Meningioma & 208 & $2(0.9 \%)$ & 210 & 0.742 & No \\
\hline Metastases & 141 & - & 141 & 0.191 & No \\
\hline Vascular arteriovenous malformation & 13 & $1(7.1 \%)$ & 14 & 0.041 & Yes \\
\hline Moyamoya disease & 12 & $3(20 \%)$ & 15 & 0.041 & Yes \\
\hline Epilepsy surgery & 11 & $1(8.3 \%)$ & 12 & 0.000 & Yes \\
\hline Total & 741 & 9 & 750 & 0.023 & Yes \\
\hline
\end{tabular}

Table 4 Number of people scrubbed and infection frequency

\begin{tabular}{|l|l|l|l|l|l|}
\hline Number of people scrubbed & Noninfected & Infected & -Value & Significance & Total \\
\hline 2 & 140 & 0 & 0.192 & No & 140 \\
\hline 3 & 253 & 4 & 0.004 & Yes & 257 \\
\hline 4 & 348 & 5 & 0.003 & Yes & 353 \\
\hline Total & 741 & 9 & & & 750 \\
\hline
\end{tabular}

Table 5 Presence of cerebrospinal fluid leak and infection frequency

\begin{tabular}{|l|l|l|l|l|l|}
\hline Cerebrospinal fluid leak & Noninfected & Infected & Total & $p$-Value & Significance \\
\hline Yes & 4 & $1(20 \%)$ & 5 & 0.000 & Yes \\
\hline No & 737 & $8(1 \%)$ & 745 & 0.752 & No \\
\hline Total & 741 & 9 & 750 & & \\
\hline
\end{tabular}

leak contributed the most to the occurrence of surgical site infections.

\section{Assessment of Risk Factors of Surgical Site Infection with Various Antibiotic Regimens}

Eight infections were seen in patients who received cefotaxime and one infection was seen in those who received amoxicillin-clavulanic acid. Superficial scalp infection (five patients) was the commonest surgical site infection, followed by bone flap osteitis (three patients), and all these infections were seen in those who received cefotaxime. No infection was seen in the group who received cefazolin. This was statistically significant. The incidence of infection depending on the antibiotic regimen is detailed in -Table 6. Methicillinresistant Staphylococcus aureus (MRSA) was the commonest organism isolated from surgical site infections (seven patients), while gram-negative bacteria were isolated in two

Table 6 Drug regimens and rate of infection: a comparative analysis

\begin{tabular}{|l|l|l|l|l|l|}
\hline Prophylactic antibiotic & Noninfected & Infected & Total & $p$-Value & Significance \\
\hline Cefotaxime & 242 & $8(3.2 \%)$ & 250 & 0.004 & Yes \\
\hline Amoxiclav & 249 & $1(0.4 \%)$ & 250 & 0.245 & No \\
\hline Cefazolin & 250 & - & 250 & 0.081 & No \\
\hline Total & 741 & 9 & 750 & & \\
\hline
\end{tabular}


patients. While all the MRSA isolates were sensitive to vancomycin and linezolid, the gram-negative isolates were sensitive to multiple drugs.

\section{Discussion}

\section{Incidence}

Surgical site infection is a preventable complication. A wide range $(0.8-6 \%)^{1}$ of surgical site infection following clean nonimplant craniotomies has been reported in the literature. This wide range of infection rates is not only due to various intrinsic risk factors present in different hospitals, but also because of lack of uniform strict criteria in basic issues, such as definition of surgical site infection. The total incidence of infection rate is $1.2 \%$ in the present study, which is equivalent to most of the recent studies following clean neurosurgical operations $^{1-8}$ in Western and Indian literature.

\section{Surgical Infections}

In the present study, nine surgical site infections were seen: scalp infections in five, bone flap osteitis in three, and meningitis in one. In total, $55 \%$ of the infections were attributable to scalp infection, 33\% to bone flap osteitis, and $11 \%$ to meningitis. Korinek et al ${ }^{1}$ showed scalp infection as the predominant surgical site infection. This study also showed scalp infection as a most common surgical site infection.

\section{Assessment of Risk Factors of Surgical Site Infections} The classical studies by Balch ${ }^{9}$ and Wright ${ }^{10}$ have identified certain factors that are believed to be associated with an increased incidence of surgical site infections. These are labeled as risk factors. Over the years, subsequent studies ${ }^{1,2}$ have confirmed some of these risk factors to be important ones but have called others into question. The various risk factors assessed in the present study are discussed in the following section.

\section{Age}

Williams et $\mathrm{al}^{11}$ and Forbes ${ }^{12}$ had found direct correlation between increasing age of the patient and increased likelihood of wound infection. Similar findings were also noted in other studies. ${ }^{13}$ However, many authors ${ }^{1,2,14-16}$ did not find any such relationship between age and incidence of infection. In the present study also, the infection rate was found to be quite uniform among the various age groups.

\section{Gender}

Korinek et $\mathrm{al}^{1}$ showed males as an independent risk factor for neurosurgical site infection after craniotomy; however, rest of the studies ${ }^{14,16}$ did not show any correlation between gender and increased likelihood of postoperative infection. The infection rate between the genders in the present study did not reach any statistical significance.

\section{Smoking}

Smoking as a risk factor influencing the development of postoperative wound infection was analyzed in the present study. There were 50 smokers, out of which only 1 developed surgical site infection. Rest of the eight patients who developed infection were nonsmokers; hence, no statistical significance was reached as in the studies done by Korinek et al. ${ }^{1}$

\section{Diabetes Mellitus}

Minchew and Cluff ${ }^{17}$ reported diabetics to be particularly prone to develop postoperative wound infection. However, Wright $^{10}$ in a large series of operations on diabetic patients did not find any infection rate. Similar observations were noted in the present study. No predilection for infection was found among 24 diabetic patients compared with nondiabetics. This could be due to proper preoperative control and effective management of diabetic status during postoperative period.

\section{Duration of Preoperative Hospital Stay}

A study by National Research Council (1964) had shown that the risk of infection is increased in those patients who spend a long time in hospital before surgery. Infection among the patients who stayed in the hospital for more than a week preoperatively was significantly higher compared with those with less than a week stay. In the present study, the infection rate in patients with less than 1 week of preoperative hospital stay was $1 \%$ compared with $1.3 \%$ among patients with more than 1 week preoperative hospital stay, which did not reach statistical significance.

\section{Surgical Diagnosis}

Korinek et $\mathrm{al}^{1}$ looked into the aspect of surgical diagnosis as a risk factor for surgical site infection. They found that the risk of infection was more following meningioma and brain metastasis surgeries, which was explained by hemostasis and closure difficulties in meningioma surgery and by immunosuppression in patients with brain metastasis. In the present study, statistically significant risk for infection was seen in arteriovenous malformation, moyamoya disease, and epilepsy surgeries. The increased incidence in arteriovenous malformation surgery can be explained due to difficult hemostasis, and in moyamoya disease, it is probably due to the particular surgeon's technique of extensive use of monopolar electrocoagulation of subcutaneous tissue following skin incision. However, statistical significance in epilepsy surgery could not be explained.

\section{Perioperative Corticosteroid Administration}

Corticosteroids are known to cause immunosuppression. However, the exact dosage and duration of steroids that lead to this effect are not clear and may be variable. A short course of perioperative steroids is mandatory in neurosurgical procedures. The influence of perioperative corticosteroids on postoperative infection is not clearly known. The existing reports in the literature are conflicting. Many studies ${ }^{10,13,18}$ did not find any significant increase in infection among the patients who received perioperative steroids. The present study showed $0.8 \%$ infection rate in patients who received 
steroids and $7.6 \%$ infection rates in those who did not receive steroids, which was statistically significant. The increase in infection rate in the nonsteroid group could not be explained.

\section{Head Shave}

The effects of full and local head shave were analyzed in this study. No infection was seen in patients who underwent local head shave. However, the sample size was too small, and hence no statistical significance could be attributed. The available literature does not show increase in infection following local head shave, ${ }^{19,20}$ and is in favor of hair clipping than shaving.

\section{Number of People Scrubbed}

In this study, it was noted that as the number of people scrubbed increased, there was statistically significant difference in infection rates. The infection rates when three or four people scrubbed were high, proving the point that more the number of people involved in surgery, the greater the chance of infection, ${ }^{21}$ as available in various literatures. Hence, there is a need to reduce the number of people involved in surgery, movement of staff members while surgery is in progress, talking during surgery, and maintaining that operating room doors be closed during surgery except during movement of staff members and equipment. These are the recommended practices for traffic patterns in the surgical suite available in the literature.

\section{Duration of Surgery}

Studies $1,10,13,14$ found linear relationship between the duration of surgery and the incidence of postoperative infection. The longer the operative time, the greater the incidence of infection. This is attributed to the increased airborne bacterial contamination of the wound and subarachnoid space. The present study also confirmed this, with less infection rate in surgeries lasting less than 4 hours. The infection rates in surgeries lasting 4 to 6 hours and more than 6 hours were highly statistically significant.

\section{Wound Closure}

The present study tried to analyze the role of wound closure, the type of closure, and suture material used in causing surgical site infections. There was no statistically significant differences seen; however, the sample size and the number of suture materials used were too small to comment on the exact role. The available literature ${ }^{22}$ does not show any difference in the incidence of surgical site infections.

\section{Presence of Cerebrospinal Fluid Leak}

In the present study, five patients had cerebrospinal leak, out of which one developed infection. The incidence of infection in patients with cerebrospinal fluid leak was $20 \%$ as against $1 \%$ in patients with no cerebrospinal fluid leak. This was statistically highly significant. Other studies ${ }^{1,14}$ have shown that the presence of cerebrospinal fluid leak is an independent risk factor for surgical site infection. This has been highlighted in the present study.

\section{Perioperative Altered Sensorium}

Some studies ${ }^{13,18}$ have noted higher infection rates among patients in altered sensorium compared with conscious patients. However, other studies ${ }^{1,14}$ did not find any statistically significant differences. In the present study also, no statistically significant difference was noted with respect to perioperative altered sensorium.

\section{Assessment of Risk Factors by Comparative Evaluation of Three Different Antibiotic Regimens}

In the present study, three different antibiotic regimens were used. Out of nine surgical site infections, eight were seen in patients who received cefotaxime as the prophylactic antimicrobial agent. Remaining one infection was seen in the patient who received amoxicillin-clavulanic acid. No infection was seen in the patients who received cefazolin. The infection rates accordingly were 3.2 and $0.4 \%$ in patients who received cefotaxime and amoxicillinclavulanic acid, respectively. This was statistically highly significant, indicating that cefazolin and amoxicillinclavulanic acid are much better prophylactic antimicrobial agents for preventing surgical site infections.

\section{Bacteriology of Surgical Site Infection}

In the present study, the most common organism isolated was MRSA followed by methicillin-resistant coagulase-negative Staphylococcus, Providencia species, and non-lactosefermenting gram-negative Bacillus. No organisms were isolated in four patients. Korinek et $\mathrm{al}^{1}$ showed MRSA as the most common organism isolated in surgical site infections. The present study also showed the same. Hence, there is a need to constantly reassess the micro-epidemiologic data of the particular institution as there can be a change in the trend of causative agents as noted in the previous NIMHANS study, which showed predominance of gram-negative organisms. ${ }^{3}$

\section{Antibiotic Sensitivity Pattern}

The antibiotic sensitivity pattern of the organisms isolated from the surgical site infections showed that the staphylococcal species, both MRSA and methicillinresistant coagulase-negative Staphylococcus, are sensitive to vancomycin and linezolid.

\section{Conclusion}

The present study has provided an insight into the incidence and risk factors contributing to surgical site infections, and the efficacious antibiotic regimen protocols. This undertaking is an effort to provide a glimpse into the status of surgical site infections in our institution. The present study has included only clean, nonimplant, fresh, elective craniotomies and is one of the few studies to compare different antibiotic regimen protocols. The future study is planned to include the remaining neurosurgical wounds, spinal surgeries, and extrasurgical site infections in 
our institution. This endeavor is like the "tip of an iceberg" phenomenon and the future study is planned in the institution to explore all aspects of postoperative infections.

\section{References}

1 Korinek AM, Golmard JL, Elcheick A, et al. Risk factors for neurosurgical site infections after craniotomy: a critical reappraisal of antibiotic prophylaxis on 4,578 patients. $\mathrm{Br} \mathrm{J}$ Neurosurg 2005;19(2):155-162

2 Korinek AM, Baugnon T, Golmard JL, van Effenterre R, Coriat P, Puybasset L. Risk factors for adult nosocomial meningitis after craniotomy: role of antibiotic prophylaxis. Neurosurgery 2006; 59(1):126-133, discussion 126-133

3 Srinivas D, Veena Kumari HB, Somanna S, Bhagavatula I, Anandappa $C B$. The incidence of postoperative meningitis in neurosurgery: an institutional experience. Neurol India 2011; 59(2):195-198

4 Sharma MS, Vohra A, Thomas P, et al. Effect of risk-stratified, protocol-based perioperative chemoprophylaxis on nosocomial infection rates in a series of 31927 consecutive neurosurgical procedures (1994-2006). Neurosurgery 2009;64(6):1123-1130, discussion 1130-1131

5 Arunodaya GR. Infections in neurology and neurosurgery intensive care units. Neurol India 2001;49(Suppl 1):S51-S59

6 Barker FG II. Efficacy of prophylactic antibiotics against meningitis after craniotomy: a meta-analysis. Neurosurgery 2007;60(5):887-894, discussion 887-894

7 Gulati S, Kapil A, Das B, Dwivedi SN, Mahapatra AK. Nosocomial infections due to Acinetobacter baumannii in a neurosurgery ICU. Neurol India 2001;49(2):134-137

8 Patir R, Mahapatra AK, Banerji AK. Risk factors in postoperative neurosurgical infection. A prospective study. Acta Neurochir (Wien) 1992;119(1-4):80-84

9 Balch RE. Wound infections complicating neurosurgical procedures. J Neurosurg 1967;26(1):41-45

10 Wright RL. A survey of possible etiologic agents in postoperative craniotomy infections. J Neurosurg 1966;25(2):125-132
11 Williams REO, McDonald JC, Blowere R. Incidence of wound infection in England and Wales: a report of the public health laboratory service. Lancet 1960;2:659-663

12 Forbes GB. Staphylococal infection of operation wounds with special reference to topical antibiotic prophylaxis. Lancet 1961; 2(7201):505-509

13 Mahapatra AK, Banerji AK, Bhatia R, Tandon PN. Prevalence of infection among neurosurgical patients: a prospective study of 507 operated patients. Neurol India 1989;37:229-237

14 Valentini LG, Casali C, Chatenoud L, Chiaffarino F, Uberti-Foppa C, Broggi G. Surgical site infections after elective neurosurgery: a survey of 1747 patients. Neurosurgery 2008;62(1):88-95, discussion 95-96

15 Reichert MC, Medeiros EA, Ferraz FA. Hospital-acquired meningitis in patients undergoing craniotomy: incidence, evolution, and risk factors. Am J Infect Control 2002;30(3): 158-164

16 van de Beek D, de Gans J, Spanjaard L, Weisfelt M, Reitsma JB, Vermeulen $M$. Clinical features and prognostic factors in adults with bacterial meningitis. N Engl J Med 2004;351(18): 1849-1859

17 Minchew BH, Cluff LE. Studies of the epidemiology of staphylococcal infection. I. Infection in hospitalized patients. J Chronic Dis 1961;13:354-373

18 Mollman HD, Haines SJ. Risk factors for postoperative neurosurgical wound infection. A case-control study. J Neurosurg 1986;64(6):902-906

19 Moiyadi AV, Sumukhi U, Shetty PM, Biswas S, Kelkar RS. Scalp flora in Indian patients undergoing craniotomy for brain tumors - Implications for pre-surgical site preparation and surgical site infection. Indian J Neurosurg 2012;1:28-32

20 Tokimura H, Tajitsu K, Tsuchiya M, et al. Cranial surgery without head shaving. J Craniomaxillofac Surg 2009;37(8):477-480

21 Pryor F, Messmer PR. The effect of traffic patterns in the OR on surgical site infections. AORN J 1998;68(4):649-660

22 Erman T, Demirhindi H, Göçer AI, Tuna M, Ildan F, Boyar B. Risk factors for surgical site infections in neurosurgery patients with antibiotic prophylaxis. Surg Neurol 2005;63(2):107-112, discussion 112-113 